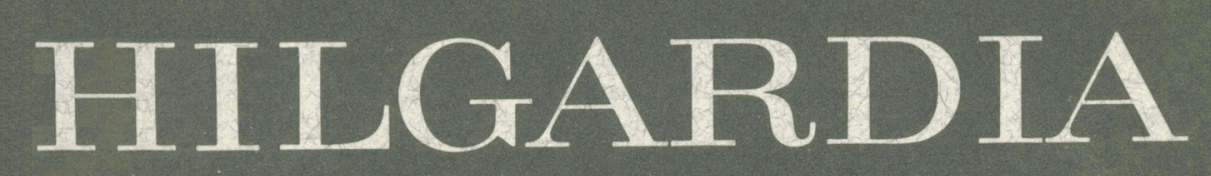

A JOURNAL OF AGRICULTURAL SCIENCE PUBLISHED BY THE CALIFORNIA AGRICULTURALEXPERIMENT STATION

Volume 53 - Number.5 August 1985

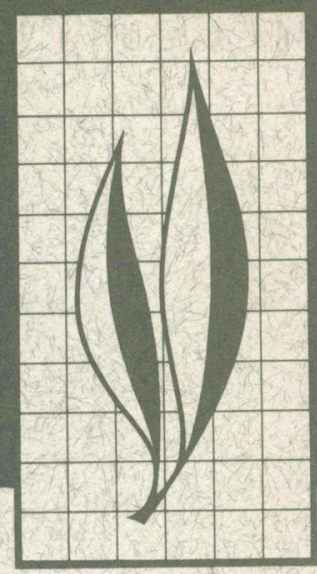

\title{
Plant-Parasitic Nematodes Associated with Forest Trees in California
}

Joyce W. Lownsbery and Benjamin F. Lownsbery 


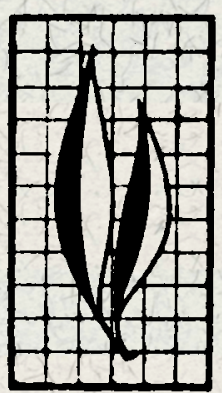

As a first step in assessing the importance of plant-parasitic nematodes to California forestry, soil and root samples were taken from 32 kinds of trees in the major forested areas of California. Ninety-seven percent of the 228 samples were from conifers, and 82 percent were from trees important in the lumber industry, mainly ponderosa and Jeffrey pines, coast redwood, Douglas fir, and red fir. In the rhizosphere of these trees, 97 described and 54 undescribed, species of plant-parasitic nematodes in $\mathbf{4 6}$ genera were found. Species varied with climate and kind of tree. Most common overall were Criconemella annulata, Xipbinema californicum, Gracilacus epacris, Pratylenchus macrostylus, Rbizonema sequoiae, Spbaeronema californicum, Trichodorus californicus, Tylencborbyncbus cylindricus, Filenchus vulgaris, Meloidogyne sp., and Ditylenchus anchilisposomus. These nematodes were often present in large numbers and it is likely that parasitism by some species constitutes one of the stresses to California forest trees.

\section{THE AUTHORS:}

Joyce W. Lownsbery is Associate in the Agricultural Experiment Station, Division of Nematology, University of California, Davis.

Benjamin F. Lownsbery is Nematologist and Professor Emeritus, Division of Nematology, University of California, Davis.

\section{ACKNOWLEDGMENTS}

The authors thank D. R. Viglierchio, D. J. Raski, E. M. Noffsinger, and T. O. Powers for their support in this project. 


\section{Plant-Parasitic Nematodes Associated with Forest Trees in California ${ }^{1}$}

\section{INTRODUCTION}

NeARly half THE LAND AREA of the state of California is forested (Lane 1984). In 1977 over 100,000 people were employed in the state's lumber, wood products, and paper industries, and the value added by manufacture of industry products was over $\$ 3$ billion (U.S. Dep. Commerce 1981). Plant-parasitic nematodes are known to reduce yields of all kinds of crop plants, including forest trees (Webster 1972), but those inhabiting California forests have received scant attention. A few pertinent observations can be gleaned from University of California and California Department of Food and Agriculture records (Siddiqui, Sher, and French 1973). The pinewood nematode, Bursapbelenchus xylophilus Steiner, has been found in isolated urban situations in Pacific Grove and Yreka, California, but has not been found in the forest, or associated with forest disease (U.S. Dep. Agric. Animal and Plant Health Insp. Serv. 1980). Effects on growth of some western forest conifer seedlings by Xiphinema bakeri, which occurs in California forests, and by other nematode parasites not known to occur there, have been reported (Maggenti and Viglierchio 1975; Viglierchio and Maggenti 1975; Viglierchio 1978; 1979). The survey reported here was conducted to determine which plant-parasitic nematodes occur naturally in the rhizosphere of California timber trees. We view this as an essential first step toward understanding the importance of nematodes to California forestry.

\section{METHODS}

Samples of soil and roots (228) were taken around 32 kinds of trees (table 1) from those floristic zones of California (Stebbins and Major, 1965; fig. 1) in which timber trees are found. The North Coast and Central Coast zones are similar. These climatic zones include the immediate coast, which seldom sees frost; has cool, wet winters; cool, dry but foggy summers; and is the home of the coast redwood and Douglas fir. The zones range to $1,000 \mathrm{~m}(3,300 \mathrm{ft})$ on the ridges of the Coast Range where winters are cold and ponderosa pines are found, and 2,700 $\mathrm{m}(9,000 \mathrm{ft})$ in the Trinity Alps with foxtail and western white pines.

The mountainous Cascade-Northern Sierra and Sierran zones are also similar, with mixed conifers, including ponderosa pine, incense cedar, white fir, and Douglas fir at $600 \mathrm{~m}$ to $2,000 \mathrm{~m}(2,000 \mathrm{ft}$ to $6,600 \mathrm{ft})$ and pure stands of red fir above $2,000 \mathrm{~m}(6,600 \mathrm{ft})$. In winter, freezing temperatures are common at lower elevations, and arctic conditions prevail near the crest of the Sierra. The Inyo zone is made up of a portion of the eastern Sierra which has severe winters, is drier than the Sierran zones mentioned above, and is the home of large stands of Jeffrey and pinyon pines.

We obtained 224 of our 228 samples from the five floristic zones just described; including samples from sea level to $3,000 \mathrm{~m}(10,000 \mathrm{ft})$. Most of the samples were from Jeffrey pine, ponderosa pine, Douglas fir, coast redwood, and red fir, important timber

${ }^{1}$ Accepted for publication April 12, 1985. 
TABLE 1. SAMPLES FROM 32 CALIFORNIA FOREST TREE SPECIES

\begin{tabular}{|c|c|c|}
\hline Latin name & Common name & $\begin{array}{l}\text { Number } \\
\text { of samples }\end{array}$ \\
\hline Abies concolor (Gord. \& Glend.) Lindl. & White fir & 9 \\
\hline Abies magnifica A. Murr & Red fir & 24 \\
\hline Betula occidentalis Hook & Water birch & 1 \\
\hline Cupressus macnabiana A. Murr. & McNab cypress & 1 \\
\hline Cupressus macrocarpa Hartw. & Monterey cypress & 2 \\
\hline Juniperus osteosperma (Torr.) Little & Utah juniper & 2 \\
\hline Libocedris decurrens Torr. & Incense cedar & 5 \\
\hline Picea sitchensis (Bong.) Carr. & Sitka spruce & 1 \\
\hline Pinus aristata Engelm. & Bristlecone pine & 1 \\
\hline Pinus attenuata Lemm. & Knobcone pine & 2 \\
\hline Pinus balfourina Grev. \& Balf. & Foxtail pine & 2 \\
\hline Pinus contorta var. murrayana (Grev. \& Balf.) Engelm. & Lodgepole pine & 4 \\
\hline Pinus coulteri D. Don & Coulter pine & 1 \\
\hline Pinus flexilis James & Limber pine & 2 \\
\hline Pinus jeffreyi Grev. \& Balf. & Jeffrey pine & 50 \\
\hline Pinus lambertiana Dougl. & Sugar pine & 3 \\
\hline Pinus monophylla Torr. \& Frem. & Singleleaf pinyon pine & 9 \\
\hline Pinus monticola Dougl. & Western white pine & 1 \\
\hline Pinus muricata D. Don & Bishop pine & 1 \\
\hline Pinus ponderosa Laws. & Ponderosa pine & 32 \\
\hline Pinus radiata $\mathrm{D}$. Don & Monterey pine & 1 \\
\hline Pinus sabiniana Dougl. & Digger pine & 2 \\
\hline Populus fremontii S. Wats. & Fremont cottonwood & 1 \\
\hline Populus tremuloides Michx. & Quaking aspen & 2 \\
\hline Pseudotsuga menziesii (Mirb.) Franco & Douglas fir & 31 \\
\hline Quercus agrifolia Nee & Coast live oak & 1 \\
\hline Quercus Kelloggi Newb. & California black oak & 2 \\
\hline Salix lasiandra Benth. & Yellow willow & 1 \\
\hline Salix lasiolepis Benth. & Arroyo willow & 1 \\
\hline Salix sp. & Willow & 1 \\
\hline Sequoia sempervirens (D. Don) Endl. & Coast redwood & 31 \\
\hline Sequoiadendron giganteum (Lindl.) Buchholz & Giant redwood & 1 \\
\hline Total & & 228 \\
\hline
\end{tabular}

species. Each sample consisted of about $4 \mathrm{~kg}(9 \mathrm{lb})$ of soil and a handful of feeder roots taken from the $10 \mathrm{~cm}$ to $40 \mathrm{~cm}$ ( 4 in to $16 \mathrm{in}$ ) depth. An effort was made to take samples in spots where there were few or no roots of plants other than the target tree. This is feasible in many California forest locations because of the open nature of the forest.

In case of delay in the nematode extraction process, samples were stored in plastic bags at $10^{\circ} \mathrm{C}\left(50^{\circ} \mathrm{F}\right)$. The soil was mixed for homogeneity and nematodes were extracted from a $250 \mathrm{cc}$ ( 1 cup) sample using Jenkins's (1964) method. The product was allowed to settle, and supernatant water was siphoned off to the $10 \mathrm{~mL}$ level. Nematodes in this $10 \mathrm{~mL}$ were killed and fixed by slow addition of $10 \mathrm{~mL}$ of a boiling solution of 4 percent formaldehyde and 1 percent glycerin, a modification of Robbins's (1978) method. This product was stored in $20 \mathrm{~mL}$ vials and numbers of each nematode species were determined by microscopic examination of aliquots. Roots were blenderized in water for 1 minute and nematodes were extracted from the product using Jenkins's (1964) method, then killed, and fixed as described above. Formaldehyde-preserved nematodes were mounted in dehydrated glycerin on 1,350 microscopic slides following Thorne's (1961) slow method. 


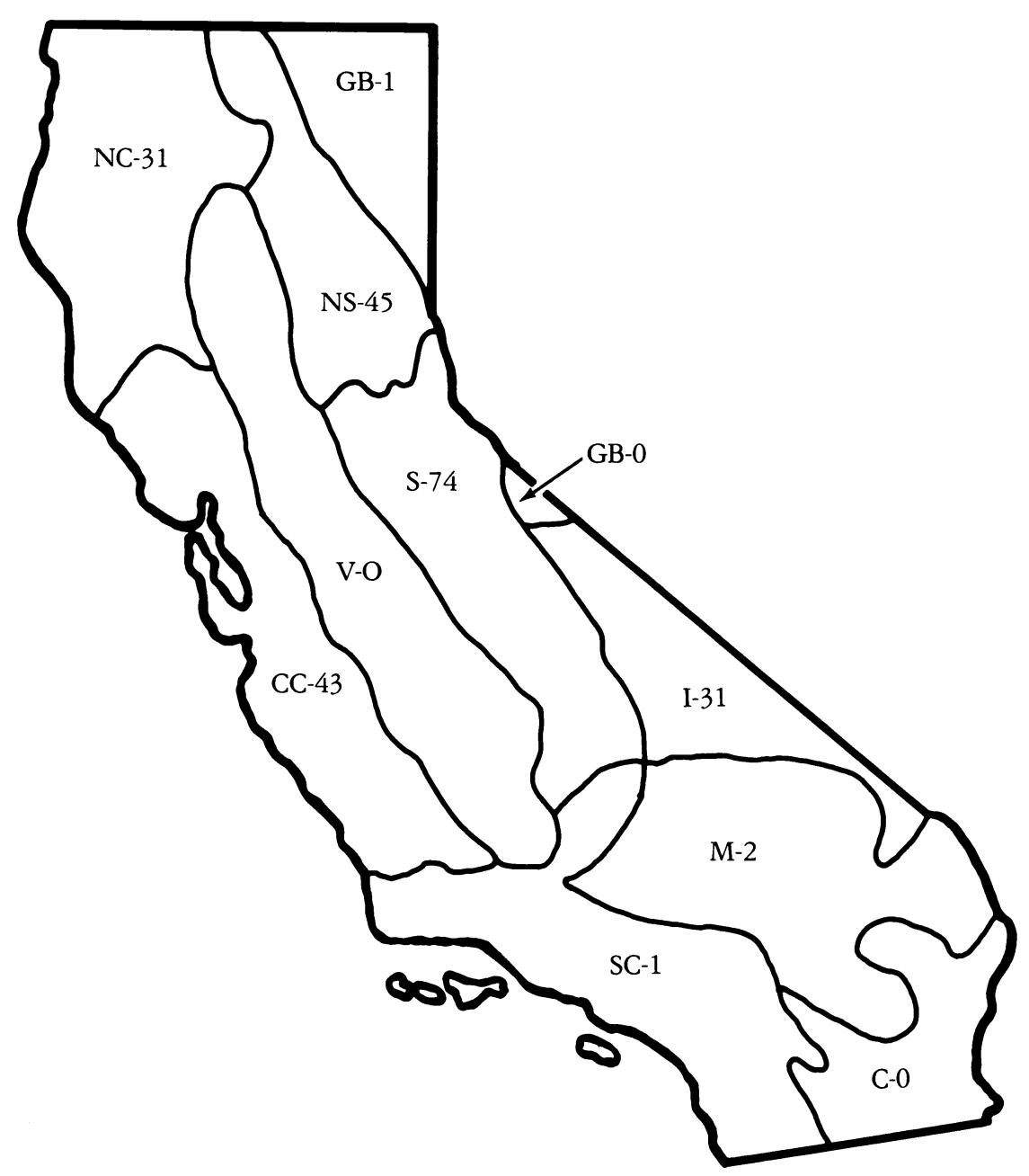

Fig. 1. Number of samples taken in each of Stebbins and Major's floristic subdivisions of California. $N C$, North Coast; NS, Cascade-North Sierra; GB, Great Basin; CC, Central Coast; V, Valley; $S$, Sierra; $I$, Inyo; $M$, Mojave; $S C$, Southern California; $C$, Colorado.

Species identifications were made using both formaldehyde-preserved and glycerinmounted specimens because certain morphological characters are seen more easily in one or the other preservative. The specimens mounted in glycerin will be placed in the University of California Davis Nematode Collection.

\section{RESULTS AND CONCLUSIONS}

The plant-parasitic nematode species found most often was the root ectoparasite Criconemella annulata (table 2). It occurred in two-thirds of the Jeffrey pine samples (table 3) and with 13 other kinds of trees, often in concentrations greater than 200 per $250 \mathrm{cc}$ (1 cup) of soil (table 4). Criconemella annulata is apparently adapted to regions with cold winters. Originally described from Montana (Taylor 1936), it has been reported on a variety of trees and shrubs from mountainous areas in California and New Mexico (Raski and Golden 1966; Raski and Riffle 1967), and in British Columbia, Alberta, and the Canadian 
TABLE 2. SAMPLES CONTAINING THE 11 MOST COMMONLY FOUND PLANT-PARASITIC NEMATODES, ALL ZONES AND TREE SPECIES COMBINED

\begin{tabular}{lc}
\hline \hline Nematode & $\mathbf{( \% )}$ \\
\hline Criconemella annulata & 43 \\
Xiphinema californica & 31 \\
Gracilacus epacris & 15 \\
Pratylenchus macrostylus & 13 \\
Rhizonema sequoiae & 13 \\
Sphaeronema californicum & 13 \\
Trichodorus californicus & 11 \\
Tylenchorbynchus cylindricus & 10 \\
Filenchus vulgaris & 9 \\
Meloidogyne sp. & 9 \\
Ditylenchus anchilisposomus & 9 \\
\hline
\end{tabular}

arctic (Wu 1965). In our study it was notably rare on coast redwood and in coastal valleys.

Xiphinema californicum was common in all zones and with nearly all tree species. This nematode has been previously identified as $X$. americanum Cobb, but all the specimens we have seen from California have the longer odontostyle and more expanded lip region of $X$. californicum (Lamberti and Bleve-Zacho 1979). Whether X. californicum is a species different from $X$. americanum or a race of it is debatable. No differences in host range or biology are known at present. Xiphinema californicum was found from sea level to $3,000 \mathrm{~m}(10,000 \mathrm{ft})$ in the Sierra, where it was present in large numbers. This apparent tolerance of a range of widely different environments seems inconsistent with the difficulties that are encountered in culturing the nematode. The culture difficulties are probably related to intolerance of rapid fluctuation in temperature and moisture (Lownsbery and Maggenti 1963) and sensitivity to low oxygen levels (Van Gundy et al. 1962), conditions more apt to obtain in culture than in nature.

Gracilacus epacris was found most frequently on the coast and on coast redwood, where it was often accompanied by Rhizonema sequoiae, Bakernema variabile, Boleodorus thylactus, and several species of Basiria. These nematodes were found in the dense mat of feeder roots that coast redwood produces in the surface layer of soil. Gracilacus epacris was originally described from a walnut orchard in the Central Valley of California (Allen and Jensen 1950). It has never been found in the Central Valley again, and may have been washed down there via streams originating in the Sierra, where it is fairly common. Rbizonema sequoiae, only recently found and described, (Cid Del Prado Vera et al. 1983) is distributed over most of the range of coast redwood, from Marin County near San Francisco to the Smith River near the Oregon border in Del Norte County. It also occurred in the Cascades-Northern Sierra and in the Sierra on ponderosa and Jeffrey pines. In past geological epochs, coast redwood grew over a much larger range than it occupies at present (Raven and Axelrod 1978). Rhizonema sequoiae may have parasitized coast redwood in its broader range, persisting now on trees that succeeded coast redwood. Boleodorus thylactus and Basiria spp. were rare or absent except in the coastal zones.

The Meloidogyne sp. often found in ponderosa and Jeffrey pine roots in the CascadeNorthern Sierra zone has perineal patterns with characteristics of both $M$. arenaria and $M$. incognita. This nematode failed to increase on either tomato or ponderosa pine in a lathhouse at Davis, California. Apparently it is a species adapted to conifers in the Sierra Nevada.

Pratylenchus macrostylus and Sphaeronema californicum were especially common with red fir in the Sierra and Cascade-Northern Sierra zones. Pratylenchus macrostylus was 
TABLE 3. PERCENTAGE OF SAMPLES

CONTAINING THE MOST COMMONLY FOUND PLANT-PARASITIC NEMATODES; FLORISTIC ZONES AND TREE SPECIES CONSIDERED SEPARATELY

\begin{tabular}{|c|c|c|c|c|}
\hline \multicolumn{5}{|c|}{ Floristic zones } \\
\hline $\begin{array}{l}\text { North } \\
\text { Coast }\end{array}$ & $\begin{array}{l}\text { Central } \\
\text { Coast }\end{array}$ & $\begin{array}{l}\text { Cascade- } \\
\text { Northern Sierra }\end{array}$ & Sierra & Inyo \\
\hline $\begin{array}{l}\text { Gracilacus } \\
\text { epacris } \\
\quad 48\end{array}$ & $\begin{array}{l}\text { Xiphinema } \\
\text { californicum } \\
44\end{array}$ & $\begin{array}{l}\text { Criconemella } \\
\text { annulata } \\
62\end{array}$ & $\begin{array}{l}\text { Criconemella } \\
\text { annulata } \\
\quad 68\end{array}$ & $\begin{array}{l}\text { Criconemella } \\
\text { annulata } \\
45\end{array}$ \\
\hline $\begin{array}{l}\text { Rbizonema } \\
\text { sequoiae } \\
\quad 45\end{array}$ & $\begin{array}{l}\text { Trichodorus } \\
\text { obscurus } \\
26\end{array}$ & $\begin{array}{l}\text { Meloidogyne } \\
\text { sp. } \\
\quad 27\end{array}$ & $\begin{array}{l}\text { Xipbinema } \\
\text { californicum } \\
30\end{array}$ & $\begin{array}{l}\text { Xipbinema } \\
\text { californicum } \\
\quad 32\end{array}$ \\
\hline $\begin{array}{l}\text { Trichodorus } \\
\text { californicus } \\
\quad 42\end{array}$ & $\begin{array}{l}\text { Gracilacus } \\
\text { epacris } \\
21\end{array}$ & $\begin{array}{l}\text { Pratylenchus } \\
\text { macrostylus } \\
18\end{array}$ & $\begin{array}{l}\text { Sphaeronema } \\
\text { californicum } \\
27\end{array}$ & $\begin{array}{l}\text { Tylenchorbynchus } \\
\text { cylindricus } \\
\quad 29\end{array}$ \\
\hline $\begin{array}{l}\text { Bakernema } \\
\text { variabile } \\
\quad 29\end{array}$ & $\begin{array}{l}\text { Trichodorus } \\
\text { californicus } \\
16\end{array}$ & $\begin{array}{c}\text { Sphaeronema } \\
\text { californicum } \\
18\end{array}$ & $\begin{array}{l}\text { Pratylenchus } \\
\text { macrostylus } \\
26\end{array}$ & $\begin{array}{l}\text { Paratylenchus } \\
\text { alleni } \\
\quad 13\end{array}$ \\
\hline $\begin{array}{l}\text { Boleodorus } \\
\text { thylactus } \\
29\end{array}$ & $\begin{array}{l}\text { Rhizonema } \\
\text { sequoiae } \\
16\end{array}$ & $\begin{array}{c}\text { Xipbinema } \\
\text { californicum } \\
18\end{array}$ & $\begin{array}{l}\text { Ditylenchus } \\
\text { anchilisposmus } \\
16\end{array}$ & $\begin{array}{l}\text { Helicotylenchus } \\
\text { clarkei } \\
\quad 10\end{array}$ \\
\hline \multicolumn{5}{|c|}{ Tree species } \\
\hline $\begin{array}{l}\text { Douglas } \\
\text { fir }\end{array}$ & $\begin{array}{l}\text { Coast } \\
\text { redwood }\end{array}$ & $\begin{array}{l}\text { Ponderosa } \\
\text { pine }\end{array}$ & $\begin{array}{l}\text { Jeffrey } \\
\text { pine }\end{array}$ & $\begin{array}{l}\text { Red } \\
\text { fir }\end{array}$ \\
\hline $\begin{array}{l}\text { Criconemella } \\
\text { annulata } \\
42\end{array}$ & $\begin{array}{l}\text { Gracilacus } \\
\text { epacris } \\
\quad 65\end{array}$ & $\begin{array}{l}\text { Criconemella } \\
\text { annulata } \\
444\end{array}$ & $\begin{array}{l}\text { Criconemella } \\
\text { annulata } \\
64\end{array}$ & $\begin{array}{l}\text { Criconemella } \\
\text { annulata } \\
75\end{array}$ \\
\hline $\begin{array}{l}\text { Xipbinema } \\
\text { californicum } \\
26\end{array}$ & $\begin{array}{l}\text { Rbizonema } \\
\text { sequoiae } \\
55\end{array}$ & $\begin{array}{l}\text { Xiphinema } \\
\text { californicum } \\
28\end{array}$ & $\begin{array}{l}\text { Xiphinema } \\
\text { californicum } \\
\quad 34\end{array}$ & $\begin{array}{l}\text { Pratylenchus } \\
\text { macrostylus } \\
\quad 42\end{array}$ \\
\hline $\begin{array}{l}\text { Trichodorus } \\
\text { californicus } \\
26\end{array}$ & $\begin{array}{l}\text { Boleodorus } \\
\text { thylactus } \\
\quad 39\end{array}$ & $\begin{array}{l}\text { Meloidogyne } \\
\text { sp. } \\
\quad 25\end{array}$ & $\begin{array}{l}\text { Meloidogyne } \\
\text { sp. } \\
\quad 22\end{array}$ & $\begin{array}{l}\text { Merlinius } \\
\text { conicus } \\
29\end{array}$ \\
\hline $\begin{array}{l}\text { Filenchus } \\
\text { vulgaris } \\
19\end{array}$ & $\begin{array}{l}\text { Trichodorus } \\
\text { californicus } \\
\quad 32\end{array}$ & $\begin{array}{l}\text { Rbizonema } \\
\text { sequoiae } \\
16\end{array}$ & $\begin{array}{l}\text { Tylenchorbynchus } \\
\text { cylindricus } \\
18\end{array}$ & $\begin{array}{l}\text { Sphaeronema } \\
\text { californicum } \\
25\end{array}$ \\
\hline $\begin{array}{c}\text { Gracilacus } \\
\text { epacris } \\
19\end{array}$ & $\begin{array}{l}\text { Xipbinema } \\
\text { californicum } \\
29\end{array}$ & $\begin{array}{c}\text { Trichodorus } \\
\text { californicus } \\
13\end{array}$ & $\begin{array}{l}\text { Sphaeronema } \\
\text { californicum } \\
16\end{array}$ & $\begin{array}{l}\text { Filenchus } \\
\text { aquilonius } \\
\quad 21\end{array}$ \\
\hline
\end{tabular}

described from Ontario and British Columbia (Wu 1971). We believe this is the first time it has been reported in California.

Trichodorus californicus and T. obscurus were the two Trichodorus species found most often, especially in the two coastal zones around a variety of trees.

Tylenchidae, including species of Filenchus, Malencbus, Tylenchus, Coslenchus, and Miculenchus, were widely distributed on many tree species. Feeding habits of these are not well known. Some feed on epidermal cells, root hairs, and mosses. Ditylenchus spp., often found in root extracts, may be mycorrhizal parasites (Riffle 1971). Aphelenchoides spp. were common, and probably include mycophagous species and associates of bark beetles. 
TABLE 4. PLANT-PARASITIC NEMATODES FOUND IN FORESTS IN SEVEN CALIFORNIA FLORISTIC ZONES (FIG. 1), AND TREE SPECIES WITH WHICH THEY WERE ASSOCIATED, BASED ON SAMPLES FROM 228 LOCATIONS

\begin{tabular}{|c|c|c|c|}
\hline $\begin{array}{l}\text { Genus and } \\
\text { described species }\end{array}$ & $\begin{array}{l}\text { Host } \\
\text { association }\end{array}$ & $\begin{array}{l}\text { Number of } \\
\text { detections }\end{array}$ & $\begin{array}{c}\text { Floristic } \\
\text { zones }\end{array}$ \\
\hline \multicolumn{4}{|l|}{ Aphelenchoides $(13)^{*}$} \\
\hline $\begin{array}{l}\text { A. cibolensis } \\
\text { Riffle }\end{array}$ & $\begin{array}{l}\text { White fir } \\
\text { Incense cedar } \\
\text { Lodgepole pine } \\
\text { Jeffrey pine } \\
\text { Ponderosa pine } \\
\text { Douglas fir }\end{array}$ & $\begin{array}{l}1 \\
1 \\
1 \\
2 \\
2 \\
1\end{array}$ & $\begin{array}{l}\text { S } \\
\text { NS } \\
\text { NS } \\
\text { NS } \\
\text { NS, S } \\
\text { S }\end{array}$ \\
\hline $\begin{array}{l}\text { A. clarus } \\
\text { Thorne and Malek }\end{array}$ & $\begin{array}{l}\text { Bristlecone pine } \\
\text { Jeffrey pine }\end{array}$ & $\begin{array}{l}1 \\
1\end{array}$ & $\begin{array}{l}\mathrm{I} \\
\mathrm{S}\end{array}$ \\
\hline $\begin{array}{l}\text { A. cyrtus } \\
\text { Paesler }\end{array}$ & $\begin{array}{l}\text { Red fir } \\
\text { Jeffrey pine } \\
\text { Ponderosa pine } \\
\text { Douglas fir } \\
\text { Coast redwood }\end{array}$ & $\begin{array}{l}1 \\
6 \\
2 \\
4 \\
2\end{array}$ & $\begin{array}{c}\text { S } \\
\text { NS, S } \\
\text { S, I } \\
\text { NC, S } \\
\text { NC, CC }\end{array}$ \\
\hline $\begin{array}{l}\text { A. saprophilus } \\
\text { Franklin }\end{array}$ & $\begin{array}{l}\text { Red fir } \\
\text { Bristlecone pine } \\
\text { Jeffrey pine } \\
\text { Ponderosa pine }\end{array}$ & $\begin{array}{l}1 \\
1 \\
1 \\
1\end{array}$ & $\begin{array}{l}\text { S } \\
\text { I } \\
\text { S } \\
\text { S }\end{array}$ \\
\hline $\begin{array}{l}\text { A. singhi } \\
\text { Das }\end{array}$ & $\begin{array}{l}\text { Bristlecone pine } \\
\text { Jeffrey pine }\end{array}$ & $\begin{array}{l}1 \\
1\end{array}$ & $\begin{array}{c}\text { I } \\
\text { NS }\end{array}$ \\
\hline $\begin{array}{l}\text { A. spinosus } \\
\text { Paesler }\end{array}$ & $\begin{array}{l}\text { McNab cypress } \\
\text { Douglas fir }\end{array}$ & $\begin{array}{l}1 \\
1\end{array}$ & $\begin{array}{l}\mathrm{NC} \\
\mathrm{NC}\end{array}$ \\
\hline \multicolumn{4}{|l|}{ Bakernema $(1)^{*}$} \\
\hline $\begin{array}{l}\text { B. variabile } \\
\text { Raski and Golden }\end{array}$ & $\begin{array}{l}\text { Ponderosa pine }{ }^{\dagger} \\
\text { Douglas fir } \\
\text { Coast redwood }{ }^{\dagger}\end{array}$ & $\begin{array}{l}1 \\
1 \\
8\end{array}$ & $\begin{array}{l}\text { NC } \\
\text { CC } \\
\text { NC }\end{array}$ \\
\hline \multicolumn{4}{|l|}{ Basiria $(5)^{*}$} \\
\hline $\begin{array}{l}\text { B. duplexa } \\
\text { (Hagemeyer and Allen) Geraert }\end{array}$ & Coast redwood & 1 & $\mathrm{NC}$ \\
\hline $\begin{array}{l}\text { B. flandriensis } \\
\text { Geraert }\end{array}$ & Coast redwood & 1 & $\mathrm{NC}$ \\
\hline $\begin{array}{l}\text { B. gracilis } \\
\text { (Thorne) Siddiqi }\end{array}$ & $\begin{array}{l}\text { Douglas fir } \\
\text { Coast redwood }\end{array}$ & $\begin{array}{l}1 \\
3\end{array}$ & $\begin{array}{c}\mathrm{CC} \\
\mathrm{NC}, \mathrm{CC}\end{array}$ \\
\hline $\begin{array}{l}\text { B. graminopbila } \\
\text { Siddiqi }\end{array}$ & Coast redwood & 1 & NC \\
\hline $\begin{array}{l}\text { Basiroides Thorne and Malek }(1)^{*} \\
\text { Boleodorus }(1)^{*}\end{array}$ & Coast redwood & 1 & NC \\
\hline $\begin{array}{l}\text { B. thylactus } \\
\text { Thorne }\end{array}$ & $\begin{array}{l}\text { Monterey cypress } \\
\text { Knobcone pine } \\
\text { Douglas fir } \\
\text { Arroyo willow } \\
\text { Coast redwood }{ }^{\dagger}\end{array}$ & $\begin{array}{r}1 \\
1 \\
1 \\
1 \\
12\end{array}$ & $\begin{array}{c}\text { CC } \\
\text { CC } \\
\text { S } \\
\text { SC } \\
\text { NC, CC }\end{array}$ \\
\hline Bursaphelenchus Fuchs $(1)^{*}$ & Jeffrey pine & 1 & $\mathrm{~S}$ \\
\hline $\begin{array}{c}\text { Coslenchus }(3)^{*} \\
\text { C. acceptus } \\
\text { Andrassy }\end{array}$ & McNab cypress & 1 & $\mathrm{CC}$ \\
\hline
\end{tabular}


TABLE 4. CONTINUED

\begin{tabular}{|c|c|c|c|}
\hline $\begin{array}{l}\text { Genus and } \\
\text { described species }\end{array}$ & $\begin{array}{l}\text { Host } \\
\text { association }\end{array}$ & $\begin{array}{l}\text { Number of } \\
\text { detections }\end{array}$ & $\begin{array}{c}\text { Floristic } \\
\text { zones }\end{array}$ \\
\hline $\begin{array}{l}\text { C. costatus } \\
\text { (de Man) Siddiqi }\end{array}$ & $\begin{array}{l}\text { Arroyo willow } \\
\text { Coast redwood }\end{array}$ & $\begin{array}{l}1 \\
1\end{array}$ & $\begin{array}{c}\mathrm{SC} \\
\mathrm{NC}, \mathrm{CC}\end{array}$ \\
\hline \multicolumn{4}{|l|}{ Criconema $(6)$} \\
\hline $\begin{array}{l}\text { C. crotaloides } \\
\text { (Cobb) Schuurmans Stekhoven } \\
\text { and Teunissen }\end{array}$ & $\begin{array}{l}\text { Knobcone pine } \\
\text { Jeffrey pine } \\
\text { Douglas fir } \\
\text { Coast redwood }\end{array}$ & $\begin{array}{l}1 \\
1 \\
1 \\
3\end{array}$ & $\begin{array}{c}\mathrm{CC} \\
\mathrm{S} \\
\mathrm{NC} \\
\mathrm{NC}, \mathrm{CC}\end{array}$ \\
\hline C. longulum Gunhold & Coast redwood & 1 & $\mathrm{CC}$ \\
\hline $\begin{array}{l}\text { C. mutabile (Taylor) } \\
\text { Raski and Luc }\end{array}$ & $\begin{array}{l}\text { Coulter pine } \\
\text { Douglas fir } \\
\text { Coast live oak } \\
\text { Coast redwood }\end{array}$ & $\begin{array}{l}1 \\
1 \\
1 \\
2\end{array}$ & $\begin{array}{l}\text { CC } \\
\text { NS } \\
\text { CC } \\
\text { CC }\end{array}$ \\
\hline $\begin{array}{l}\text { C. psammophilum } \\
\text { (Krnjaic and Loof) } \\
\text { Raski and Luc }\end{array}$ & $\begin{array}{l}\text { Douglas fir } \\
\text { Coast redwood }\end{array}$ & $\begin{array}{l}1 \\
2\end{array}$ & $\begin{array}{c}\mathrm{CC} \\
\mathrm{CC}, \mathrm{NC}\end{array}$ \\
\hline $\begin{array}{l}\text { C. thornei } \\
\text { (Knobloch and Bird) } \\
\text { Raski and Luc }\end{array}$ & $\begin{array}{l}\text { White fir } \\
\text { Jeffrey pine } \\
\text { Ponderosa pine }\end{array}$ & $\begin{array}{l}1 \\
2 \\
1\end{array}$ & $\begin{array}{l}\text { NS } \\
\text { NS } \\
\text { S }\end{array}$ \\
\hline \multicolumn{4}{|l|}{ Criconemella $(6)^{*}$} \\
\hline $\begin{array}{l}\text { C. annulata (Taylor) } \\
\text { Luc and Raski }\end{array}$ & $\begin{array}{l}\text { White fir }{ }^{\dagger} \\
\text { Red fir }^{\dagger} \\
\text { Water birch } \\
\text { Utah juniper } \\
\text { Incense cedar } \\
\text { Lodgepole pine } \\
\text { Jeffrey pine }{ }^{\dagger} \\
\text { Sugar pine } \\
\text { Western white pine } \\
\text { Ponderosa pine } \\
\text { Douglas fir } \\
\text { California black oak } \\
\text { Coast redwood } \\
\text { Giant redwood }\end{array}$ & $\begin{array}{r}7 \\
18 \\
1 \\
1 \\
4 \\
1 \\
32 \\
3 \\
1 \\
14 \\
13 \\
1 \\
1 \\
1\end{array}$ & $\begin{array}{c}\text { NS, S } \\
\text { NS, S } \\
\text { I } \\
\text { I } \\
\text { S } \\
\text { S } \\
\text { NS, S, I } \\
\text { NS, S } \\
\text { S } \\
\text { S, NS, CC } \\
\text { S, NS, CC } \\
\text { I } \\
\text { CC } \\
\text { S }\end{array}$ \\
\hline $\begin{array}{l}\text { C. macrodora (Taylor) } \\
\text { Luc and Raski }\end{array}$ & $\begin{array}{l}\text { Knobcone pine } \\
\text { Ponderosa pine } \\
\text { Douglas fir } \\
\text { Coast redwood }{ }^{\dagger}\end{array}$ & $\begin{array}{l}1 \\
1 \\
2 \\
1\end{array}$ & $\begin{array}{l}\mathrm{CC} \\
\mathrm{NC} \\
\mathrm{NC} \\
\mathrm{CC}\end{array}$ \\
\hline $\begin{array}{l}\text { C. pseudobercyniensis } \\
\text { (DeGrisse and Koen) } \\
\text { Luc and Raski }\end{array}$ & Willow & 1 & $S$ \\
\hline $\begin{array}{l}\text { C. teres (Raski) } \\
\text { Luc and Raski }\end{array}$ & $\begin{array}{l}\text { Ponderosa pine } \\
\text { Coast redwood }\end{array}$ & $\begin{array}{l}2 \\
3\end{array}$ & $\begin{array}{l}\text { NS, S } \\
\text { CC }\end{array}$ \\
\hline $\begin{array}{l}\text { C. vernus (Raski and Golden) } \\
\text { Luc and Raski }\end{array}$ & Yellow willow & 1 & I \\
\hline $\begin{array}{l}\text { C. xenoplax (Raski) } \\
\text { Luc and Raski }\end{array}$ & $\begin{array}{l}\text { Ponderosa pine } \\
\text { Montery pine } \\
\text { Coast redwood }\end{array}$ & $\begin{array}{l}1 \\
1 \\
1\end{array}$ & $\begin{array}{l}\mathrm{CC} \\
\mathrm{CC} \\
\mathrm{CC}\end{array}$ \\
\hline $\begin{array}{l}\text { Crossonema }(1)^{*} \\
\text { C. venustum } \\
\text { Mehta and Raski }\end{array}$ & Ponderosa pine & 1 & $\mathrm{CC}$ \\
\hline
\end{tabular}


TABLE 4. CONTINUED

\begin{tabular}{|c|c|c|c|}
\hline $\begin{array}{l}\text { Genus and } \\
\text { described species }\end{array}$ & $\begin{array}{l}\text { Host } \\
\text { association }\end{array}$ & $\begin{array}{l}\text { Number of } \\
\text { detections }\end{array}$ & $\begin{array}{l}\text { Floristic } \\
\text { zones }\end{array}$ \\
\hline \multicolumn{4}{|l|}{ Deladenus $(1)^{*}$} \\
\hline $\begin{array}{l}\text { D. durus } \\
\text { (Cobb) Thorne }\end{array}$ & Coast redwood & 1 & NC \\
\hline Diphtborophora de $\operatorname{Man}(1)^{*}$ & $\begin{array}{l}\text { Knobcone pine } \\
\text { Jeffrey pine } \\
\text { Sugar pine } \\
\text { Singleleaf pinyon pine } \\
\text { Bishop pine } \\
\text { Ponderosa pine } \\
\text { Douglas fir }{ }^{\dagger} \\
\text { Coast redwood }\end{array}$ & $\begin{array}{l}1 \\
1 \\
1 \\
1 \\
1 \\
2 \\
4 \\
6\end{array}$ & $\begin{array}{c}\text { CC } \\
\text { NS } \\
\text { NS } \\
\text { I } \\
\text { CC } \\
\text { NS } \\
\text { NC, CC, S } \\
\text { NC, CC }\end{array}$ \\
\hline \multicolumn{4}{|l|}{ Ditylenchus $(13)^{*}$} \\
\hline $\begin{array}{l}\text { D. anchilisposomus } \\
\text { (Tarjan) Fortuner }\end{array}$ & $\begin{array}{l}\text { Red fir } \\
\text { McNab cypress } \\
\text { Incense cedar } \\
\text { Limber pine } \\
\text { Jeffrey pine } \\
\text { Ponderosa pine } \\
\text { Douglas fir } \\
\text { Coast redwood }\end{array}$ & $\begin{array}{l}3 \\
1 \\
1 \\
1 \\
5 \\
4 \\
1 \\
4\end{array}$ & $\begin{array}{c}\text { S } \\
\text { CC } \\
\text { S } \\
\text { S } \\
\text { NS, S } \\
\text { S } \\
\text { S } \\
\text { NC, CC }\end{array}$ \\
\hline $\begin{array}{l}\text { D. convallariae } \\
\text { Sturhan and Friedman }\end{array}$ & Coast redwood & 1 & $\mathrm{C}$ \\
\hline D. destructor Thorne & White fir & 1 & NS \\
\hline $\begin{array}{l}\text { D. lutonensis } \\
\text { (Siddiqi) Fortuner }\end{array}$ & Red fir & 1 & $S$ \\
\hline $\begin{array}{l}\text { D. myceliophagus } \\
\text { Goodey }\end{array}$ & $\begin{array}{l}\text { Red fir } \\
\text { Incense cedar } \\
\text { Jeffrey pine } \\
\text { Ponderosa pine } \\
\text { Douglas fir }\end{array}$ & $\begin{array}{l}1 \\
1 \\
4 \\
1 \\
1\end{array}$ & $\begin{array}{c}\text { S } \\
\text { S } \\
\text { NS, S } \\
\text { NS } \\
\text { CC }\end{array}$ \\
\hline $\begin{array}{l}\text { D. tenuidens } \\
\text { Gritsenko }\end{array}$ & Coast redwood & 1 & $\mathrm{CC}$ \\
\hline $\begin{array}{l}\text { D. triformis } \\
\text { Hirschmann and Sasser }\end{array}$ & $\begin{array}{l}\text { Knobcone pine } \\
\text { Jeffrey pine } \\
\text { Douglas fir } \\
\text { Yellow willow }\end{array}$ & $\begin{array}{l}1 \\
3 \\
1 \\
1\end{array}$ & $\begin{array}{l}\mathrm{I} \\
\mathrm{S} \\
\mathrm{CC} \\
\mathrm{SC}\end{array}$ \\
\hline \multicolumn{4}{|l|}{ Filenchus $(20)^{*}$} \\
\hline $\begin{array}{l}\text { F. andrassyi } \\
\text { (Szczygiel) Andrassy }\end{array}$ & Coast redwood & 1 & NC \\
\hline $\begin{array}{l}\text { F. aquilonius } \\
\text { (Wu) comb. } \mathrm{n} \text {. }\end{array}$ & $\begin{array}{l}\text { Red fir } \\
\text { Jeffrey pine } \\
\text { Sugar pine } \\
\text { Ponderosa pine } \\
\text { Quaking aspen } \\
\text { Douglas fir }\end{array}$ & $\begin{array}{l}7 \\
5 \\
1 \\
1 \\
1 \\
1\end{array}$ & $\begin{array}{c}\text { NC, NS, S } \\
\text { NS, S, I } \\
\text { S } \\
\text { S } \\
\text { S } \\
\text { S }\end{array}$ \\
\hline $\begin{array}{l}\text { F. mirus } \\
\text { (Husain and Khan) comb. } \mathrm{n} .\end{array}$ & $\begin{array}{l}\text { Douglas fir } \\
\text { Coast redwood }\end{array}$ & $\begin{array}{l}1 \\
1\end{array}$ & $\begin{array}{l}\mathrm{NC} \\
\mathrm{NC}\end{array}$ \\
\hline $\begin{array}{l}\text { F. plattensis } \\
\text { (Thorne \& Malek) Niblack and Bernard }\end{array}$ & $\begin{array}{l}\text { Red fir } \\
\text { Ponderosa pine } \\
\text { Douglas fir }\end{array}$ & $\begin{array}{l}2 \\
1 \\
1\end{array}$ & $\begin{array}{c}\mathrm{S} \\
\mathrm{S} \\
\mathrm{NC}\end{array}$ \\
\hline
\end{tabular}


TABLE 4. CONTINUED

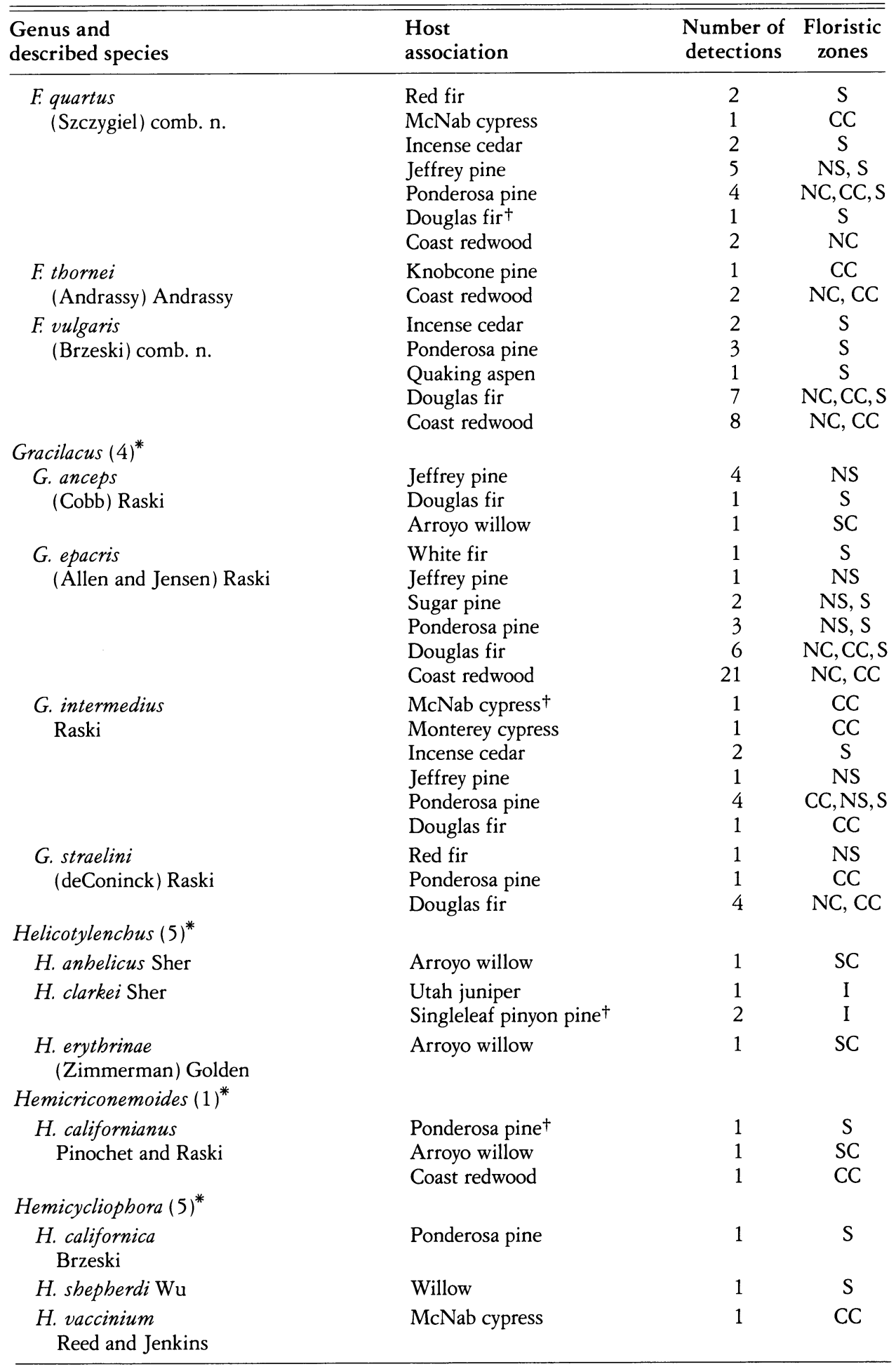


TABLE 4. CONTINUED

\begin{tabular}{|c|c|c|c|}
\hline $\begin{array}{l}\text { Genus and } \\
\text { described species }\end{array}$ & $\begin{array}{l}\text { Host } \\
\text { association }\end{array}$ & $\begin{array}{l}\text { Number of } \\
\text { detections }\end{array}$ & $\begin{array}{c}\text { Floristic } \\
\text { zones }\end{array}$ \\
\hline Hexatylus Goodey $(1)^{*}$ & Jeffrey pine & 1 & NS \\
\hline \multicolumn{4}{|l|}{ Hoplolaimus $(1)^{*}$} \\
\hline H. californicus & Monterey cypress & 1 & $\mathrm{CC}$ \\
\hline Sher & Arroyo willow & 1 & SC \\
\hline Hoplotylus s'Jacob $(1)^{*}$ & Douglas fir & 3 & $\mathrm{NC}, \mathrm{CC}$ \\
\hline \multicolumn{4}{|l|}{ Longidorus $(1)^{*}$} \\
\hline $\begin{array}{l}\text { L. vineacola } \\
\text { Sturhan and Weischer }\end{array}$ & Singleleaf pinyon pine & 1 & $S$ \\
\hline \multicolumn{4}{|l|}{ Malenchus $(6)^{*}$} \\
\hline $\begin{array}{l}\text { M. acaryensis } \\
\text { Andrassy }\end{array}$ & Knobcone pine & 1 & $\mathrm{CC}$ \\
\hline Meloidogyne Chitwood (1)* & White fir & 2 & NS, S \\
\hline & Jeffrey pine ${ }^{\dagger}$ & 10 & NS, S \\
\hline & Ponderosa pine ${ }^{\dagger}$ & 8 & NS, S \\
\hline & Yellow willow & 1 & I \\
\hline \multicolumn{4}{|l|}{ Merlinius $(5)^{*}$} \\
\hline M. conicus & White fir & 1 & NS \\
\hline (Allen) Siddiqi & Red fir ${ }^{\dagger}$ & 7 & $S$ \\
\hline & Foxtail pine ${ }^{\dagger}$ & 2 & $\mathrm{~s}$ \\
\hline & Jeffrey pine & 6 & NS, S, I \\
\hline & Singleaf pinyon pine & 1 & I \\
\hline & Ponderosa pine & 2 & NS, S \\
\hline $\begin{array}{l}\text { M. grandis } \\
\text { (Allen) Siddiqi }\end{array}$ & Singleleaf pinyon pine & 1 & $M$ \\
\hline $\begin{array}{l}\text { M. lineatus } \\
\text { (Allen) Siddiqi }\end{array}$ & Jeffrey pine & 1 & NS \\
\hline $\begin{array}{l}\text { M. microdorus } \\
\text { (Geraert) Siddiqi }\end{array}$ & Jeffrey pine & 1 & NS \\
\hline M. notbus & White fir & 2 & NS \\
\hline (Allen) Siddiqi & Jeffrey pine & 1 & NS \\
\hline \multicolumn{4}{|l|}{ Miculenchus $(1)^{*}$} \\
\hline M. salvus & Douglas fir & 1 & NC \\
\hline Andrassy & Coast redwood & 2 & NC \\
\hline \multicolumn{4}{|l|}{ Nagelus $(1)^{*}$} \\
\hline $\begin{array}{l}\text { N. leptus } \\
\quad \text { (Allen) Siddiqi }\end{array}$ & Willow & 1 & $S$ \\
\hline \multicolumn{4}{|l|}{ Neodolichodorus (1)* } \\
\hline N. obtusus & McNab cypress & 1 & $\mathrm{CC}$ \\
\hline (Allen) Andrassy & Digger pine & 1 & $\mathrm{CC}$ \\
\hline \multicolumn{4}{|l|}{ Neopsilenchus $(2)^{*}$} \\
\hline N. magnidens (Thorne) & Douglas fir & 2 & $\mathrm{NC}, \mathrm{CC}$ \\
\hline Thorne and Malek & Coast redwood & 4 & $\mathrm{NC}, \mathrm{CC}$ \\
\hline \multicolumn{4}{|l|}{ Notbotylenchus $(3)^{*}$} \\
\hline \multirow[t]{3}{*}{ N. acris Thorne } & Sitka spruce & 1 & NC \\
\hline & Jeffrey pine & 1 & $\mathrm{~S}$ \\
\hline & Coast redwood & 1 & NC \\
\hline
\end{tabular}


TABLE 4. CONTINUED

\begin{tabular}{|c|c|c|c|}
\hline $\begin{array}{l}\text { Genus and } \\
\text { described species }\end{array}$ & $\begin{array}{l}\text { Host } \\
\text { association }\end{array}$ & $\begin{array}{l}\text { Number of } \\
\text { detections }\end{array}$ & $\begin{array}{c}\text { Floristic } \\
\text { zones }\end{array}$ \\
\hline \multicolumn{4}{|l|}{ Ottolenchus $(3)^{*}$} \\
\hline $\begin{array}{l}\text { O. facultativus } \\
\text { (Szczygiel) Brzeski }\end{array}$ & $\begin{array}{l}\text { White fir } \\
\text { Red fir } \\
\text { McNab cypress } \\
\text { Incense cedar } \\
\text { Jeffrey pine } \\
\text { Ponderosa pine } \\
\text { Douglas fir } \\
\text { Arroyo willow } \\
\text { Coast redwood }\end{array}$ & $\begin{array}{l}1 \\
1 \\
1 \\
1 \\
2 \\
1 \\
2 \\
1 \\
1\end{array}$ & $\begin{array}{l}\text { NS } \\
\text { S } \\
\text { CC } \\
\text { S } \\
\text { NS, I } \\
\text { NS } \\
\text { NC } \\
\text { SC } \\
\text { CC }\end{array}$ \\
\hline $\begin{array}{l}\text { O. belenae } \\
\text { (Szczygiel) Brzeski }\end{array}$ & $\begin{array}{l}\text { Red fir } \\
\text { Coast redwood }\end{array}$ & $\begin{array}{l}1 \\
1\end{array}$ & $\begin{array}{c}\mathrm{S} \\
\mathrm{NC}\end{array}$ \\
\hline Paraphelenchus Micoletzky (1)* & $\begin{array}{l}\text { White fir } \\
\text { Red fir } \\
\text { Incense cedar } \\
\text { Jeffrey pine } \\
\text { Sugar pine } \\
\text { Ponderosa pine } \\
\text { Douglas fir }\end{array}$ & $\begin{array}{l}1 \\
2 \\
1 \\
6 \\
1 \\
3 \\
3\end{array}$ & $\begin{array}{l}\text { NS } \\
\text { S } \\
\text { S } \\
\text { NS, S } \\
\text { S } \\
\text { S } \\
\text { S }\end{array}$ \\
\hline \multicolumn{4}{|l|}{ Pararotylenchus $(3)^{*}$} \\
\hline $\begin{array}{l}\text { P. blothrotylus } \\
\text { Baldwin and Bell }\end{array}$ & Singleleaf pinyon pine ${ }^{\dagger}$ & 1 & M \\
\hline $\begin{array}{l}\text { P. truncocephalus } \\
\text { Baldwin and Bell }\end{array}$ & $\begin{array}{l}\text { White fir } \\
\text { Jeffrey pine } \\
\text { Quaking aspen }\end{array}$ & $\begin{array}{l}1 \\
1 \\
1\end{array}$ & $\begin{array}{l}\text { NS } \\
\text { NS } \\
\text { S }\end{array}$ \\
\hline $\begin{array}{l}\text { Paratrichodorus Siddiqi }(1)^{*} \\
\text { Paratylenchus }(2)^{*}\end{array}$ & \multicolumn{3}{|c|}{ Paratylenchus $(2)^{*}$} \\
\hline $\begin{array}{l}\text { P. alleni } \\
\text { Raski }\end{array}$ & $\begin{array}{l}\text { Utah juniper }{ }^{\dagger} \\
\text { Bristlecone pine } \\
\text { Jeffrey pine }\end{array}$ & $\begin{array}{l}1 \\
1 \\
2\end{array}$ & $\begin{array}{l}\text { I } \\
\text { I } \\
\text { I }\end{array}$ \\
\hline $\begin{array}{l}\text { P. neoamblycephalus } \\
\text { Geraert }\end{array}$ & $\begin{array}{l}\text { Water birch }{ }^{\dagger} \\
\text { Ponderosa pine }\end{array}$ & $\begin{array}{l}1 \\
1\end{array}$ & $\begin{array}{l}\text { I } \\
\text { NS }\end{array}$ \\
\hline \multicolumn{4}{|l|}{ Pratylenchoides $(2)^{*}$} \\
\hline $\begin{array}{l}\text { P. magnicauda } \\
\text { (Thorne) Baldwin, Luc and Bell } \\
\text { Pratylenchus }(4)^{*}\end{array}$ & $\begin{array}{l}\text { Quaking aspen } \\
\text { Yellow willow }\end{array}$ & $\begin{array}{l}1 \\
1\end{array}$ & $\begin{array}{l}S \\
S\end{array}$ \\
\hline $\begin{array}{l}\text { P. macrostylus } \\
\mathrm{Wu}\end{array}$ & $\begin{array}{l}\text { White fir } \\
\text { Red fir } \\
\text { Incense cedar } \\
\text { Jeffrey pine } \\
\text { Sugar pine } \\
\text { Ponderosa pine } \\
\text { Quaking aspen } \\
\text { Douglas fir } \\
\text { Giant redwood }\end{array}$ & $\begin{array}{r}5 \\
12 \\
2 \\
4 \\
1 \\
2 \\
1 \\
1 \\
1\end{array}$ & $\begin{array}{c}\text { NS, S } \\
\text { NS, S } \\
\text { S } \\
\text { NS, S, I } \\
\text { S } \\
\text { S } \\
\text { S } \\
\text { S } \\
\text { S }\end{array}$ \\
\hline $\begin{array}{l}\text { P. penetrans (Cobb) } \\
\text { Filipjev and Schuurmans Stekhoven }\end{array}$ & $\begin{array}{l}\text { Ponderosa pine } \\
\text { Douglas fir }\end{array}$ & $\begin{array}{l}1 \\
1\end{array}$ & $\begin{array}{l}\mathrm{CC} \\
\mathrm{CC}\end{array}$ \\
\hline $\begin{array}{l}\text { P. sefaensis } \\
\text { Fortuner }\end{array}$ & Jeffrey pine & 2 & $\mathrm{~S}$ \\
\hline
\end{tabular}


TABLE 4. CONTINUED

\begin{tabular}{|c|c|c|c|}
\hline $\begin{array}{l}\text { Genus and } \\
\text { described species }\end{array}$ & $\begin{array}{l}\text { Host } \\
\text { association }\end{array}$ & $\begin{array}{l}\text { Number of } \\
\text { detections }\end{array}$ & $\begin{array}{c}\text { Floristic } \\
\text { zones }\end{array}$ \\
\hline \multicolumn{4}{|l|}{ Pseudbalenchus (1)* } \\
\hline $\begin{array}{l}\text { P. minutus } \\
\text { Tarjan }\end{array}$ & $\begin{array}{l}\text { Jeffrey pine } \\
\text { Douglas fir }\end{array}$ & $\begin{array}{l}1 \\
1\end{array}$ & $\begin{array}{c}\mathrm{NC} \\
\mathrm{S}\end{array}$ \\
\hline \multicolumn{4}{|l|}{ Rbizonema $(1)^{*}$} \\
\hline $\begin{array}{l}\text { R. sequoiae } \\
\text { Cid del Prado Vera, Lownsbery, } \\
\text { and Maggenti }\end{array}$ & $\begin{array}{l}\text { Jeffrey pine } \\
\text { Ponderosa pine } \\
\text { Douglas fir } \\
\text { Coast redwood }\end{array}$ & $\begin{array}{r}2 \\
5 \\
5 \\
17\end{array}$ & $\begin{array}{c}\text { NS } \\
\text { NS, S } \\
\text { NC, CC } \\
\text { NC, CC }\end{array}$ \\
\hline Rotylenchus Filipjev $(2)^{*}$ & $\begin{array}{l}\text { Jeffrey pine } \\
\text { Ponderosa pine } \\
\text { Douglas fir }\end{array}$ & $\begin{array}{l}1 \\
1 \\
2\end{array}$ & $\begin{array}{l}\text { NS } \\
\text { NS } \\
\text { NS }\end{array}$ \\
\hline \multicolumn{4}{|l|}{ Scutylencbus $(1)^{*}$} \\
\hline $\begin{array}{l}\text { S. tesselatus } \\
\text { (Goodey) Siddiqi }\end{array}$ & $\begin{array}{l}\text { Jeffrey pine } \\
\text { Yellow willow } \\
\text { Willow }\end{array}$ & $\begin{array}{l}1 \\
1 \\
1\end{array}$ & $\begin{array}{l}\text { NS } \\
\text { I } \\
\text { S }\end{array}$ \\
\hline \multicolumn{4}{|l|}{ Sphaeronema $(1)^{*}$} \\
\hline $\begin{array}{l}\text { S. californicum } \\
\text { Raski and Sher }\end{array}$ & $\begin{array}{l}\text { White fir } \\
\text { Red fir } \\
\text { Jeffrey pine } \\
\text { Sugar pine } \\
\text { Ponderosa pine } \\
\text { Douglas fir } \\
\text { Willow } \\
\text { Coast redwood } \\
\text { Giant redwood }\end{array}$ & $\begin{array}{l}1 \\
6 \\
9 \\
1 \\
7 \\
2 \\
1 \\
1 \\
1\end{array}$ & $\begin{array}{l}\text { NS } \\
\text { S } \\
\text { NS, S } \\
\text { S } \\
\text { NS, S } \\
\text { S } \\
\text { S } \\
\text { CC } \\
\text { S }\end{array}$ \\
\hline \multicolumn{4}{|l|}{ Trichodorus $(7)^{*}$} \\
\hline $\begin{array}{l}\text { T. aequalis } \\
\text { Allen }\end{array}$ & $\begin{array}{l}\text { Jeffrey pine }{ }^{\dagger} \\
\text { Ponderosa pine }\end{array}$ & $\begin{array}{l}2 \\
2\end{array}$ & $\begin{array}{l}\mathrm{I} \\
\mathrm{S}\end{array}$ \\
\hline $\begin{array}{l}\text { T. californicus } \\
\text { Allen }\end{array}$ & $\begin{array}{l}\text { Sitka spruce } \\
\text { Ponderosa pine } \\
\text { Douglas fir }{ }^{\dagger} \\
\text { Yellow willow } \\
\text { Coast redwood } \\
\text { Giant redwood }\end{array}$ & $\begin{array}{r}1 \\
4 \\
8 \\
1 \\
10 \\
1\end{array}$ & $\begin{array}{c}\text { NC } \\
\text { NC, S } \\
\text { NC, CC, NS } \\
\text { SC } \\
\text { NC, CC } \\
\text { S }\end{array}$ \\
\hline $\begin{array}{l}\text { T. intermedius } \\
\text { Rodriguez M. and Bell }\end{array}$ & Yellow willow & 1 & SC \\
\hline $\begin{array}{l}\text { T. obscurus } \\
\text { Allen }\end{array}$ & $\begin{array}{l}\text { McNab cypress } \\
\text { Monterey cypress } \\
\text { Jeffrey pine } \\
\text { Ponderosa pine } \\
\text { Digger pine } \\
\text { Douglas fir }{ }^{\dagger} \\
\text { Coast redwood }\end{array}$ & $\begin{array}{l}1 \\
1 \\
1 \\
2 \\
1 \\
5 \\
5\end{array}$ & $\begin{array}{c}\mathrm{CC} \\
\mathrm{CC} \\
\mathrm{NS} \\
\mathrm{CC} \\
\mathrm{CC} \\
\mathrm{NC}, \mathrm{CC} \\
\mathrm{NC}, \mathrm{CC}\end{array}$ \\
\hline $\begin{array}{l}\text { T. sparsus } \\
\text { Szczygiel }\end{array}$ & $\begin{array}{l}\text { Douglas fir } \\
\text { Coast redwood }\end{array}$ & $\begin{array}{l}1 \\
1\end{array}$ & $\begin{array}{l}\mathrm{NC} \\
\mathrm{NC}\end{array}$ \\
\hline \multicolumn{4}{|l|}{ Tylenchorbynchus $(1)^{*}$} \\
\hline $\begin{array}{l}\text { T. cylindricus } \\
\text { Cobb }\end{array}$ & $\begin{array}{l}\text { Utah juniper } \\
\text { Bristlecone pine }{ }^{\dagger} \\
\text { Foxtail pine }\end{array}$ & $\begin{array}{l}1 \\
1 \\
1\end{array}$ & $\begin{array}{l}\text { I } \\
\text { I } \\
\text { S }\end{array}$ \\
\hline
\end{tabular}


TABLE 4. CONTINUED

\begin{tabular}{|c|c|c|c|}
\hline $\begin{array}{l}\text { Genus and } \\
\text { described species }\end{array}$ & $\begin{array}{l}\text { Host } \\
\text { association }\end{array}$ & $\begin{array}{l}\text { Number of } \\
\text { detections }\end{array}$ & $\begin{array}{l}\text { Floristic } \\
\text { zones }\end{array}$ \\
\hline & Jeffrey pine & 9 & S, I \\
\hline & Singleleaf pinyon pine ${ }^{\dagger}$ & 6 & $\mathrm{~S}, \mathrm{I}, \mathrm{M}$ \\
\hline & Ponderosa pine & 1 & $\mathrm{~S}$ \\
\hline & California black oak & 2 & I \\
\hline & Yellow willow & 1 & SC \\
\hline \multicolumn{4}{|l|}{ Tylenchus $(7)^{*}$} \\
\hline $\begin{array}{l}\text { T. butteus } \\
\text { Thorne and Malek }\end{array}$ & Douglas fir & 1 & NC \\
\hline T. ditissimus & Bristlecone pine & 1 & I \\
\hline \multirow[t]{3}{*}{ Brzeski } & Jeffrey pine & 1 & $\mathrm{~S}$ \\
\hline & Douglas fir & 2 & $\mathrm{~S}$ \\
\hline & Coast redwood & 1 & NC \\
\hline T. maius & Red fir & 1 & NS \\
\hline \multirow{2}{*}{ Andrassy } & Jeffrey pine & 2 & NS, S \\
\hline & Ponderosa pine & 1 & $\mathrm{~S}$ \\
\hline T. sandneri & White fir & 1 & NS \\
\hline \multirow[t]{2}{*}{ Wasilewska } & Jeffrey pine & 1 & S \\
\hline & Douglas fir & 1 & $S$ \\
\hline \multicolumn{4}{|l|}{ Xipbinema $(2)^{*}$} \\
\hline \multirow{4}{*}{$\begin{array}{l}X . \text { bakeri } \\
\text { Williams }\end{array}$} & Ponderosa pine & 2 & $S$ \\
\hline & Fremont cottonwood & 1 & I \\
\hline & Quaking aspen & 1 & $\mathrm{~S}$ \\
\hline & Coast redwood & 1 & NC \\
\hline \multirow{19}{*}{$\begin{array}{l}X . \text { californicum } \\
\text { Lamberti and Bleve-Zacheo }\end{array}$} & White fir & 3 & NS, S \\
\hline & McNab cypress & 1 & $\mathrm{CC}$ \\
\hline & Monterey cypress & 2 & $\mathrm{CC}$ \\
\hline & Utah juniper & 1 & I \\
\hline & Incense cedar & 2 & $\mathrm{~S}$ \\
\hline & Knobcone pine & 1 & $\mathrm{CC}$ \\
\hline & Coulter pine & 1 & $\mathrm{CC}$ \\
\hline & Limber pine ${ }^{\dagger}$ & 1 & $\mathrm{~S}$ \\
\hline & Jeffrey pine & 17 & NS, S, I, GB \\
\hline & Singleleaf pinyon pine & 3 & $\mathrm{I}, \mathrm{M}$ \\
\hline & Western white pine & 1 & $\mathrm{~S}$ \\
\hline & Ponderosa pine & 9 & CC, NS, S \\
\hline & Monterey pine & 1 & $\mathrm{CC}$ \\
\hline & Digger pine & 1 & CC \\
\hline & Quaking aspen & 1 & $\mathrm{~S}$ \\
\hline & Douglas fir & 8 & NC, CC, NS, S \\
\hline & California black oak & 2 & I \\
\hline & Yellow willow & 1 & SC \\
\hline & Coast redwood & 9 & $\mathrm{NC}, \mathrm{CC}$ \\
\hline
\end{tabular}

${ }^{*}$ Number of species (in parentheses).

†Nematode species found with that specific host at a concentration of 200 or more per $250 \mathrm{cc}$ of soil. 
In general, nematodes common in California agriculture (Siddiqui, Sher, and French 1973) were not found in California forests. Exceptions to this were Xiphinema californicum and Tylenchorbynchus cylindricus, which we found frequently, and Criconemella xenoplax and Paratylenchus neoamblycephalus, found very infrequently. We did not find many of the plant-parasitic nematodes reported in forests in the eastern United States, or in other parts of the world (Ruehle 1967; Kiryanova and Krall 1980). Many of these reports concerned land that had been in agricultural crops at some time, or had a climate not much different from nearby agricultural areas. With the exception of some coastal and foothill apple orchards and vineyards, California forest has never been in agriculture and has a climate greatly different from agricultural areas.

These California forest trees have been exposed to their nematode parasites for more than a million years, a time sufficient to achieve a balance allowing survival of host and parasite. This does not mean that the trees would not grow better if relieved of their burden of nematode parasites. Nematodes may be one of the agents that is weakening trees and predisposing them to attack by bark beetles. At present there is no practical way to reduce nematode populations in standing forests. We can protect nursery seedlings and avoid distributing nematodes by application of a soil fumigant before planting, and this is advisable. California foresters select seed from most vigorous trees for propagation in nurseries. They may be selecting indirectly for nematode tolerance by this process. Common occurrence of the virus vector Xiphinema californicum in all zones is of interest. Virus diseases are not known to be important in conifers, but Fulton (1969) has transmitted tobacco ringspot virus to roots of Cupressus arizonica. 


\section{LITERATURE CITED}

ALLEN, M. W., and H. J. JENSEN

1950. Cacopaurus epacris, new species (Nematoda: Criconematidae) a nematode parasite of California black walnut roots. Proc. Helminthol. Soc. Wash. 17:10-14.

CID DEL PRADO VERA, I., B. F. LOWNSBERY, and A. R. MAGGENTI

1983. Rhizonema sequoiae n.gen. n.sp. from Coast Redwood Sequoia sempervirens (D. Don) Endl. J. Nematol. 15:460-67.

FULTON, J. P.

1969. Transmission of tobacco ringspot virus to the roots of a conifer by a nematode. Phytopathology 59:236.

JENKINS, W. R.

1964. A rapid centrifugal-flotation technique for separating nematodes from soil. Plant Dis. Reptr. 48:692.

KIRYANOVA, E. S., and E. L. KRALL

1980. Plant-parasitic nematodes and their control. Vol. II. Academy of Sciences, USSR. Inst. Zool. (English translation). New Delhi, India: Amerind. Publ. Co. 748 pp.

LAMBERTI, F., and T. BLEVE-ZACHEO

1979. Studies of Xiphinema americanum sensu lato with descriptions of fifteen new species (Nematoda, Longidoridae). Nematol. Medit. 7:51-106.

LANE, H. V., ed.

1984. The world almanac and book of facts. New York: Newspaper Enterprize Assoc. 928 pp.

LOWNSBERY, B. F, and A. R. MAGGENTI

1963. Some effects of soil temperature and soil moisture on population levels of Xiphinema americanum. Phytopathology 53:667-68.

MAGGENTI, A. R., and D. R. VIGLIERCHIO

1975. Sequoia sempervirens and Sequoiadendron giganteum: hosts of common plant-parasitic nematodes of California. Plant Dis. Reptr. 59:116-19.

RASKI, D. J., and A. M. GOLDEN

1966. Studies on the genus Criconemoides Taylor, 1936 with descriptions of eleven new species and Bakernema variabile n.sp. (Criconematidae: Nematoda). Nematologica 11:501-65.

RASKI, D. J., and J. W. RIFFLE

1967. Two new species and further notes on Criconemoides Taylor, 1936 (Criconematidae: Nematoda). Proc. Helminthol. Soc. Wash. 34:212-19.

RAVEN, P. H., and D. I. AXELROD

1978. Origin and relationships of the California flora. Univ. Calif. Publ. in Bot. 72:1-134.

RIFFLE, J. W.

1971. Mycorrhizae 8. Effect of nematodes on root-inhabiting fungi. Proc. First North American Conf. on Mycorrhizae, April 1969. Misc. Publ. 1189. U.S. Dep. Agric. For. Serv. 97-113.

ROBBINS, R. T.

1978. A new Ataloderinae (Nematoda: Heteroderidae), Thecavermiculatus gracililancea n.gen. n.sp. J. Nematol. 10:250-54.

RUEHLE, J. L.

1967. Distribution of plant-parasitic nematodes associated with forest trees of the world. Southeastern Forest Experiment Station. U.S. Dep. Agric. For. Serv. 156 pp.

SIDDIQUI, I. A., S. A. SHER, and A. M. FRENCH

1973. Distribution of plant-parasitic nematodes in California. Calif. Dep. Food and Agric. Div. Plant Ind. $324 \mathrm{pp}$.

STEBBINS, G. L., and J. MAJOR

1965. Endemism and speciation in the California flora. Ecol. Monogr. 35:1-35.

TAYLOR, A. L.

1936. The genera and species of the Criconematinae, a subfamily of the Anguillulidae (Nematoda). Trans. Amer. Microsc. Soc. 55:391-421.

THORNE, G.

1961. Principles of Nematol. New York: McGraw Hill. 553 pp.

U.S. DEP. AGRIC. ANIMAL AND PLANT HEALTH INSP. SERV. (Compiler)

1980. Cooperative Plant Pest Rept. 5:267-84.

U.S. DEP. COMMERCE, BUREAU OF THE CENSUS

1981. 1977 Census of manufacturers. Vol. 3. Geographic area statistics, Part 1. General summary. California, pp. 5-1 to 5-88.

VAN GUNDY, S. D., L. H. STOLZY, T. E. SZUSZKIEWICS, and R. L. RACKHAM

1962. Influence of oxygen supply on survival of plant-parasitic nematodes in soil. Phytopathology 52:628-32. 
VIGLIERCHIO, D. R.

1978. Stylet-bearing nemas and growth of ponderosa pine seedlings. Forest Sci. 24:222-27.

1979. Response of Pinus ponderosa seedlings to stylet-bearing nematodes. J. Nematol. 11:377-87.

VIGLIERCHIO, D. R., and A. R. MAGGENTI

1975. Susceptibility of western forest conifers to common agricultural plant-parasitic nematodes. Plant Dis. Reptr. 59:326-28.

WEBSTER, J. M., ed.

1972. Economic nematology. New York: Academic Press. 563 pp.

WU, L.

1965. Five new species of Criconemoides Taylor, 1936 (Criconematidae: Nematoda) from Canada. Can. J. Zool. 43:203-14.

1971. Pratylenchus macrostylus n.sp. (Pratylenchinae: Nematoda). Can. J. Zool. 49:487-89.

The University of California in compliance with the Civil Rights Act of 1964, Title IX of the Education Amendments of 1972. and the Rehabilitation Act of 1973 does not discriminate on the basis of race, creed, religion, color, national origin, sex, or mental or physical handicap in any of its programs or activities, or with respect to any of its employment policies, practices, c: procedures. The University of California does not discriminate on the basis of age, ancestry, sexual orientation, marital status, citizenship, nor because individuals are disabled or Vietnam era veterans. Inquiries regarding this policy may be directed to the Affirmative Action Officer, Division of Agriculture and Natural Resources, 2120 University Ave., University of California, Berkeley, California 94720 (415) 644-4270. 